\title{
Second window ICG predicts gross-total resection and progression-free survival during brain metastasis surgery
}

\author{
Clare W. Teng, BA, ${ }^{1,2}$ Steve S. Cho, MD, MTR, ${ }^{1,2}$ Yash Singh, BS, ${ }^{1}$ Emma De Ravin, BS, ${ }^{1,2}$ \\ Keren Somers, BS, ${ }^{1}$ Love Buch, BS, ${ }^{1}$ Steven Brem, MD, ${ }^{1}$ Sunil Singhal, MD, ${ }^{3}$ \\ Edward J. Delikatny, PhD, ${ }^{4}$ and John Y. K. Lee, MD, MSCE ${ }^{1}$
}

\begin{abstract}
${ }^{1}$ Department of Neurosurgery, Hospital of the University of Pennsylvania; ${ }^{2}$ Perelman School of Medicine at the University of Pennsylvania; ${ }^{3}$ Department of Surgery, Hospital of the University of Pennsylvania; and ${ }^{4}$ Department of Radiology, University of Pennsylvania, Philadelphia, Pennsylvania
\end{abstract}

\begin{abstract}
OBJECTIVE Metastases are the most common intracranial malignancies and complete resection can provide relief of neurological symptoms and reduce recurrence. The authors' prospective pilot study in 2017 demonstrated promising results for the application of high-dose, delayed imaging of indocyanine green (ICG), known as second window ICG (SWIG), in patients undergoing surgery for brain metastases. In this prospective cohort study, the authors evaluated intraoperative imaging and clinical outcomes of treatment using SWIG.
\end{abstract}

METHODS Patients were prospectively enrolled in an approved study of high-dose, delayed ICG (SWIG) and received $5 \mathrm{mg} / \mathrm{kg}$ (2014-2018) or $2.5 \mathrm{mg} / \mathrm{kg}$ (2018-2019) ICG 24 hours preoperatively. Intraoperatively, near-infrared (NIR) imaging was performed using a dedicated NIR exoscope. NIR images were analyzed and the signal-to-background ratio (SBR) was calculated to quantify fluorescence. Residual fluorescence on the postresection NIR view was compared and correlated to the residual gadolinium enhancement on postoperative MRI. Patient survival and predictive factors were analyzed.

RESULTS In total, 51 intracranial metastases were surgically treated in 47 patients in this cohort. All 51 metastatic tumors demonstrated strong NIR fluorescence (mean SBR 4.9). In tumors $\leq 10 \mathrm{~mm}$ from the cortical surface, SWIG with $5 \mathrm{mg} / \mathrm{kg}$ ICG produced enhanced transdural tumor visibility $(91.3 \%)$ compared to $2.5 \mathrm{mg} / \mathrm{kg}(52.9 \% ; p=0.0047)$. Neoplastic margin detection using NIR fluorescence compared to white light improved sensitivity, albeit lowered specificity; however, increasing the SBR cutoff for positive fluorescence significantly improved specificity without sacrificing sensitivity, increasing the overall accuracy from $57.5 \%$ to $72.5 \%$. A lack of residual NIR fluorescence after resection was closely correlated with a lack of residual enhancement on postoperative MRI $(p=0.007)$. Among the 16 patients in whom tumor recurred at the site of surgery, postoperative MRI successfully predicted 8 cases, whereas the postresection NIR view predicted 12 cases. Progression-free survival rate at 12 months was greater for patients without residual NIR fluorescence (38\%) than for those without residual enhancement on postoperative MRI (29\%).

CONCLUSIONS The current study demonstrates the clinical benefits of the SWIG technique in surgery for patients with brain metastases. Specifically, this technique allows for dose-dependent, transdural localization of neoplasms and improved sensitivity in neoplastic margin detection. Postresection residual fluorescence can be a powerful tool to evaluate extent of resection in conjunction with MRI, and it may guide decisions on brain metastasis management.

https://thejns.org/doi/abs/10.3171/2020.8.JNS201810

KEYWORDS near-infrared fluorescence; second window indocyanine green; SWIG; brain metastasis; postoperative $\mathrm{MRI}$; recurrence; oncology

$\mathrm{B}$ RAIN metastases (BMs) are the most common malignancies in the central nervous system, as $8 \%-$ $10 \%$ of all newly diagnosed cancers metastasize to the brain..$^{1-3}$ Lung cancer, breast cancer, and melano- ma are the most common sources, collectively accounting for $67 \%-80 \%$ of BMs. ${ }^{4,5}$ A major cause of morbidity and mortality in BMs is their high local recurrence rate. Prior to the adoption of postoperative radiation therapy,

ABBREVIATIONS BM = brain metastasis; FGS = fluorescence-guided surgery; GTR = gross-total resection; ICG = indocyanine green; IR = infrared; NIR = near-IR; NPV = negative predictive value; $\mathrm{OS}=$ overall survival; $\mathrm{PFS}=$ progression-free survival; $\mathrm{PpIX}=$ protoporphyrin $I X ; \mathrm{PPV}=$ positive predictive value; $\mathrm{ROC}=$ receiver operating characteristic; SBR = signal-to-background ratio; SRS = stereotactic radiosurgery; SWIG = second window ICG; WBRT = whole-brain radiotherapy; WL = white light; 5-ALA $=5$-aminolevulinic acid.

SUBMITTED June 2, 2020. ACCEPTED August 3, 2020.

INCLUDE WHEN CITING Published online March 2, 2021; DOI: 10.3171/2020.8.JNS201810. 
local recurrence after resection reached 50\%. ${ }^{6}$ Recently, the Congress of Neurological Surgeons recommended the use of stereotactic radiosurgery (SRS) after solitary BM resection to reduce local recurrence (level 3 evidence). ${ }^{7}$ However, even with radiotherapy, local recurrence rates are still close to $10 \%$, leading to a median survival of 3 to 27 months. ${ }^{1,5,8-10}$ Furthermore, radiotherapy involves considerable risks; local radionecrosis develops in close to half of treated lesions, leading to new-onset neurological complications in up to $32 \%$ of patients. ${ }^{11-13}$

Another approach to prevent recurrence is to increase the extent of resection. Burt et al. reported that complete resection, rather than the stage of the primary lesion, determines survival in patients undergoing resection of BMs from non-small cell lung cancer. ${ }^{14}$ Importantly, a randomized clinical trial found that while SRS was associated with improved early local control of treated lesions, surgical resection led to lower risk of recurrence after 9 months..$^{15}$ To address these needs, a number of intraoperative technologies have emerged to improve resection of intracranial malignancies, among which fluorescenceguided surgery (FGS) is gradually gaining favor.

In neurosurgery, FGS research has largely focused on gliomas. This can be largely attributed to the first successful human trial of FGS with 5-aminolevulinic acid (5-ALA), a fluorophore approved by the FDA in 2017 for high-grade gliomas. Despite the success of 5-ALA in gliomas, its utility in intracranial metastasis remains contentious. Past studies have reported that more than one-third of BMs cannot be visualized with 5-ALA, with variable accumulation of protoporphyrin IX (PpIX) in different histologies. ${ }^{16-22}$ Another fluorophore that has been used to visualize BM is fluorescein. Early studies have demonstrated a sensitivity above $90 \%$ but a low specificity, and no outcome studies have been conducted..$^{23-25}$ Of note, both 5-ALA and fluorescein are visible-light spectrum fluorophores that are limited by low tissue penetration and high background autofluorescence.

Our prospective pilot study in 2017 demonstrated promising results for the application of high-dose, delayed imaging of indocyanine green (ICG), an FDA-approved near-infrared (NIR) fluorophore (excitation $805 \mathrm{~nm}$, emission $835 \mathrm{~nm}$ ) in BM surgery. This second window ICG (SWIG) technique involves intravenous infusion of highdose ICG approximately 24 hours before surgery, allowing ICG to accumulate in neoplastic tissue through the enhanced permeability and retention effect. All 13 BMs demonstrated high NIR fluorescence with a mean signalto-background ratio (SBR) of $6.62 .{ }^{10}$ We have recently closed this prospective trial; here, we report the final results demonstrating the intraoperative imaging characteristics and clinical outcomes for fluorescence-guided BM surgery using SWIG.

\section{Methods \\ Study Patients}

Patient enrollment began in October 2014. All adult patients ( $\geq 18$ years old) undergoing craniotomy for brain tumor were eligible for the trial. The main exclusion criteria included pregnancy and shellfish/iodine allergy.
The study, registered under clinicaltrials.gov (identifier: NCT03262636), was approved by the University of Pennsylvania Institutional Review Board. Patients were informed that the findings from NIR imaging would not impact surgeons' decisions on the scope of surgery. All patients gave informed consent.

\section{NIR Imaging Procedure}

Patients enrolled before April 2018 were infused intravenously with $5 \mathrm{mg} / \mathrm{kg}$ ICG $\left(\mathrm{C}_{43} \mathrm{H}_{47} \mathrm{~N}_{2} \mathrm{O}_{6} \mathrm{~S}_{2} \cdot \mathrm{Na}\right.$; Akorn Pharmaceuticals). After April 2018, the dose was decreased to $2.5 \mathrm{mg} / \mathrm{kg}$ as part of a planned dose de-escalation protocol. All infusions were conducted at an outpatient infusion center over a 1-hour period the day before surgery. In the operating room, all cases were imaged using the VisionSense iridium exoscope system with an $805-\mathrm{nm}$ laser excitation source and 820- to 860-nm emission filter; this system has demonstrated remarkable NIR sensitivity in prior studies. ${ }^{26,27}$ Real-time videos were captured in $720 \mathrm{p}$ resolution.

\section{Surgical Procedure and Image Acquisition}

Patients underwent anesthesia and craniotomy using anatomical landmarks and neuronavigation imaging. After craniotomy and before durotomy, the NIR imaging system was sterilely draped and positioned above the surgical field. Imaging was first performed at this time and referred to as "dura view." After durotomy, "cortex view" imaging was performed. Surgery then proceeded in the standard manner. "Tumor view" imaging was acquired after complete exposure of the gross tumor and before removal from the resection cavity. Areas that appeared neoplastic under white light (WL) were resected regardless of NIR fluorescence, although the presence or absence of NIR fluorescence in those areas was recorded prior to surgical removal. Additional biopsy specimens were taken if considered safe by the senior neurosurgeon. Histopathological diagnosis was performed by neuropathologists on the separate specimens and served as the gold standard for tissue identity. At the end of surgery, a "final view" of the operative field was captured. A case video illustrating the surgical procedure and image acquisition is included in Video 1.

VIDEO 1. Surgical video illustrating SWIG-guided resection of an intracranial metastasis in the left parietal lobe. NIR fluorescence was visualized using pseudocolor overlay. Copyright John Y. K. Lee. Used with permission. Click here to view.

\section{NIR Image Analysis}

The ability to localize gross tumor using the dura view and cortex view was determined by independent reviewers. This analysis was limited to tumors $\leq 10 \mathrm{~mm}$ deep, given NIR photon tissue penetration limits. ImageJ (https://imagej.nih.gov/ij/; National Institutes of Health) was used to calculate the SBR of each specimen. A region of interest was drawn by surrounding the border of the signal from pathology on the infrared (IR) image, and measurement of the mean gray value was recorded as "signal." "Background" was determined by selecting the area of normal brain parenchyma in view (Fig. 1). Upon inspection of the 

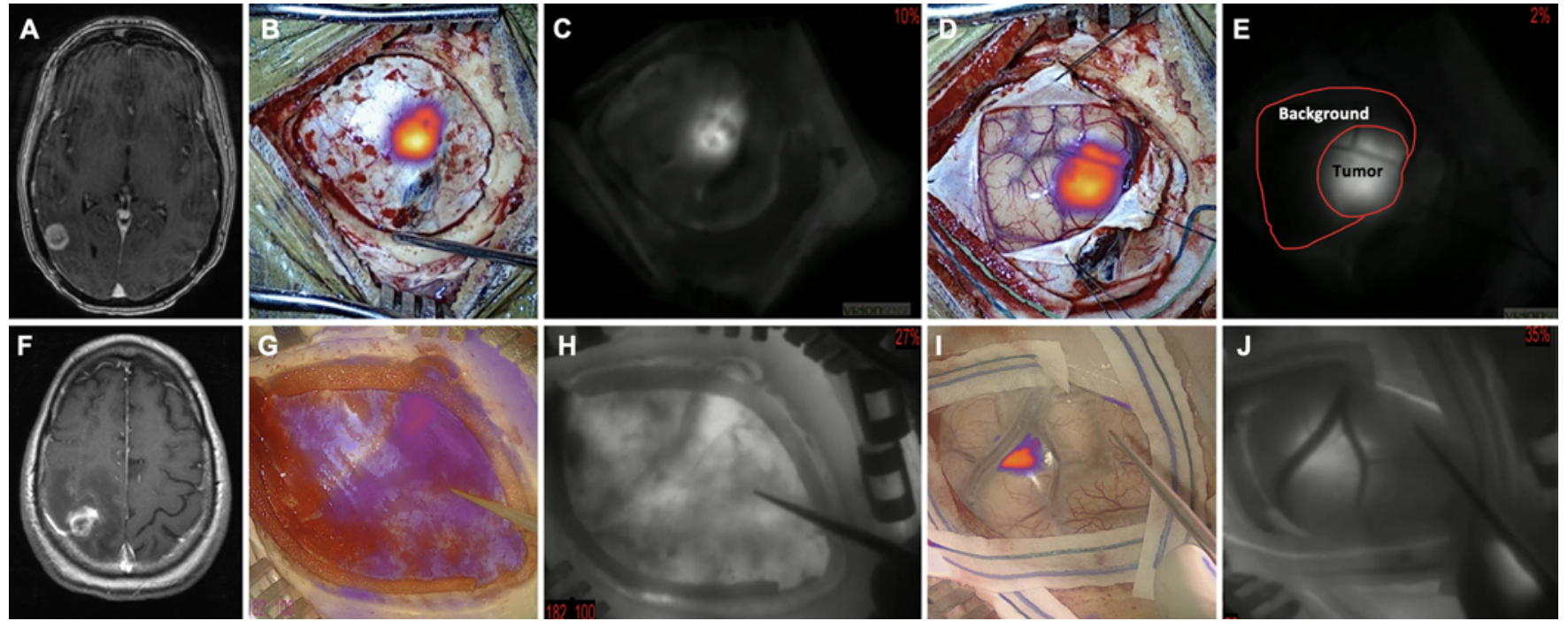

FIG. 1. Examples of successful (5 mg/kg) and unsuccessful $(2.5 \mathrm{mg} / \mathrm{kg})$ tumor localization through dura/cortex. A-E: The patient was infused intravenously with $5 \mathrm{mg} / \mathrm{kg}$ ICG approximately 24 hours before surgery. T1 MRI was performed with gadolinium at 1-mm resolution. The axial image $(\mathbf{A})$ demonstrated a contrast-enhancing superficial mass extending to the cortex, consistent with a metastasis. Intraoperatively, after craniotomy and prior to dura opening, NIR fluorescence was visualized in both pseudocolor overlay (B) and black and white (C), demonstrating successful visualization of the tumor through the intact dura. After durotomy, the NIR cortex view was imaged (D and E) and the localization of tumor on the dura view and cortex view was consistent (E). Demonstration of the method of SBR calculation: signal $=92.7$, background $=9.2, S B R=10.1$. F-J: The patient was infused intravenously with $2.5 \mathrm{mg} / \mathrm{kg} \mathrm{ICG}$ approximately 24 hours before surgery. T1 MRI was performed with gadolinium at 1-mm resolution. The axial image $(\mathbf{F})$ demonstrated a contrast-enhancing superficial mass extending to the cortex, consistent with a metastasis. Intraoperatively, after craniotomy and prior to dura opening, the NIR fluorescence image was visualized ( $\mathbf{G}$ and $\mathbf{H})$. The location of the tumor was unable to be determined solely based on the dura view. After durotomy, the NIR cortex view was visualized. In this case, the location of the tumor was successfully visualized on the cortex view. Some nonspecific signal from the skull is seen in the lower-left corner (I and $\mathrm{J}$ ). Figure is available in color online only.

final view, if residual NIR fluorescence was noted in or around the surgical cavity, the anatomical location of the fluorescence was determined (i.e., anterior/posterior cavity, deep cavity) in order to compare this location to the location of any residual gadolinium enhancement on postoperative MRI.

\section{Evaluation of Postoperative MRI}

Two postoperative MRI scans (day 1 and day 30) were performed on a 1.5-Tesla MR system using either 1-mm or 5-mm slices per hospital protocol. The images were reviewed by neuroradiologists blinded to the NIR imaging results. Locations of residual gadolinium enhancement were described on radiology reports. Gross-total resection (GTR) was defined as the absence of nodular enhancing foci near the surgical cavity within 1 month of surgery. Patients received regular follow-ups and serial MRI scans to track recurrence (a new lesion was one that was not present on prior scans and was visible in a minimum of 2 projections) or progression of the tumor ( $>20 \%$ increase in the sum of the longest distance relative to the nadir) as defined by the Response Assessment in Neuro-Oncology (RANO)-BM criteria. ${ }^{28}$

\section{Clinical Data Collection}

Patient clinical data were retrospectively collected until April 2020. Potential prognostic factors (i.e., age, sex, and primary pathology) were collected. Two patients were excluded from survival analysis due to transfer of care elsewhere immediately postsurgery. Overall survival (OS) was calculated from the date of surgery until death or until April 2020 for living patients. Progression-free survival (PFS) was calculated from the surgery date until recurrence/progression of the tumor at the resection site, death in those without radiological evidence of progression, or until April 2020 for those alive without progression.

\section{Statistical Analysis}

Stata 10 (StataCorp LLC) was used for statistical analysis. An independent t-test was used for comparing values between groups. Receiver operating characteristic (ROC) analysis was performed to evaluate diagnostic ability. Two-by-two contingency tables were constructed to calculate the sensitivity, specificity, positive predictive value (PPV), and negative predictive value (NPV). Kaplan-Meier curves of PFS and OS were constructed, and apparent differences in survival time were evaluated using a log-rank test. For all analyses, a p value $\leq 0.05$ determined statistical significance.

\section{Results}

\section{Patient Characteristics}

Between October 2014 and May 2019, 54 patients with intraparenchymal metastatic brain tumors undergoing surgical resection were consecutively enrolled in this pro- 
TABLE 1. Clinical data summary

\begin{tabular}{|c|c|}
\hline & Value \\
\hline Total no. of patients & 47 \\
\hline Total no. of metastases & 51 \\
\hline Total no. of specimens & 91 \\
\hline Age, yrs, median (range) & $61(36-82)$ \\
\hline \multicolumn{2}{|l|}{ Sex } \\
\hline Female & $34(72 \%)$ \\
\hline Male & $13(28 \%)$ \\
\hline \multicolumn{2}{|l|}{ ICG dose, $\mathrm{mg} / \mathrm{kg}$} \\
\hline 5 & $26(55 \%)$ \\
\hline 2.5 & $21(45 \%)$ \\
\hline $\begin{array}{l}\text { Time from ICG infusion to visualization, hrs, } \\
\text { mean (range) }\end{array}$ & $23.3(15.9-29.2)$ \\
\hline Prior op & $6(13 \%)$ \\
\hline Prior radiation & $18(38 \%)$ \\
\hline \multicolumn{2}{|l|}{ No. of metastases per patient } \\
\hline Single & $39(83 \%)$ \\
\hline Multiple & $8(17 \%)$ \\
\hline \multicolumn{2}{|l|}{ Tumor size, largest diameter, in $\mathrm{mm}$} \\
\hline Mean & 28.1 \\
\hline Median (range) & $24(13-69)$ \\
\hline \multicolumn{2}{|l|}{ Tumor laterality } \\
\hline Lt & $21(45 \%)$ \\
\hline Rt & $22(47 \%)$ \\
\hline Bilat & $4(9 \%)$ \\
\hline \multicolumn{2}{|l|}{ Tumor location } \\
\hline Frontal & $18(35 \%)$ \\
\hline Parietal & $11(22 \%)$ \\
\hline Temporal & $7(14 \%)$ \\
\hline Occipital & $11(22 \%)$ \\
\hline Cerebellar & $4(8 \%)$ \\
\hline \multicolumn{2}{|l|}{ Primary pathology } \\
\hline Lung cancer & $20(43 \%)$ \\
\hline Breast cancer & $9(19 \%)$ \\
\hline Melanoma & $6(13 \%)$ \\
\hline Colon cancer & $3(6 \%)$ \\
\hline $\begin{array}{l}\text { Others (ovarian, renal, prostate, endocervical, } \\
\text { esophageal) }\end{array}$ & $9(19 \%)$ \\
\hline \multicolumn{2}{|l|}{ Postop radiation therapy } \\
\hline SRS & $39(83 \%)$ \\
\hline WBRT & $2(4 \%)$ \\
\hline No radiation & $6(13 \%)$ \\
\hline
\end{tabular}

Values are presented as number (\%) of patients unless otherwise indicated.

spective trial. Seven patients were excluded (5 endoscopic cases and 2 cases with technical difficulties during image acquisition). A total of 47 patients were included in the final analysis ( 26 cases with $5 \mathrm{mg} / \mathrm{kg} \mathrm{ICG}$ and 21 with 2.5 $\mathrm{mg} / \mathrm{kg} \mathrm{ICG)}$. All patients tolerated the infusion well without major adverse events. Detailed clinical characteristics are summarized in Table 1.
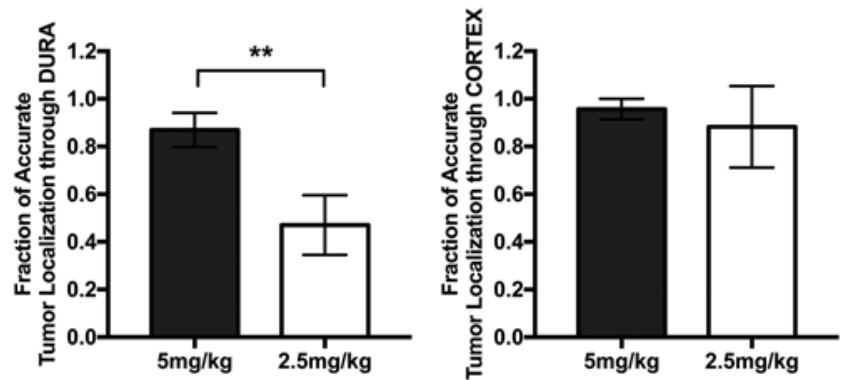

FIG. 2. Fractions of tumor accurately localized through the dura and cortex. Left: NIR signal on dura view was evaluated for its ability to guide localization of the tumor. The localization was deemed successful by the independent reviewer if the location of the tumor determined on the dura view corresponded with the location shown on the tumor view. A statistically significant difference in the fraction of accurate tumor localization through dura between the two dose groups was demonstrated $\left({ }^{* *} p=0.0047\right)$. Bars represent the standard error. Right: Postdurotomy, a similar analysis was performed on the cortex view. The difference between the two groups was not statistically significant $(p=$ 0.39). Bars represent the standard error.

\section{Tumor Localization Using NIR Fluorescence}

Prior to dura opening, NIR imaging was performed; $76.9 \%(30 / 39)$ of the tumors that were $\leq 10 \mathrm{~mm}$ below the cortex were successfully visualized through the intact dura (Fig. 1). The deepest tumor visualized in our study was $13.9 \mathrm{~mm}$. When stratified by dose, transdural localization was accomplished in $91.7 \%(22 / 24)$ in the $5-\mathrm{mg} /$ $\mathrm{kg}$ group and $53.3 \%(8 / 15)$ in the $2.5-\mathrm{mg} / \mathrm{kg}$ group $(\mathrm{p}=$ 0.0048 ), demonstrating enhanced transdural visibility in the higher dose (Fig. 2 left). Through the cortex, 94.9\% (37/39) of the tumors demonstrated visible NIR fluorescence, and there was no significant difference between the two doses: $95.8 \%(23 / 24)$ in the $5-\mathrm{mg} / \mathrm{kg}$ cases versus $93.3 \%(14 / 15)$ in the $2.5-\mathrm{mg} / \mathrm{kg}$ cases $(\mathrm{p}=0.74)$ (Fig. 2 right).

\section{Detailed Analysis of Fluorescence Characteristics}

In total, 51 distinct $\mathrm{BMs}$ were resected from $47 \mathrm{pa}$ tients. In direct view of the NIR camera, all tumors demonstrated fluorescence with an average SBR of $4.9 \pm 2.9$. Univariate linear regression analysis of tumor SBR with independent variables yielded the following results. The $5-\mathrm{mg} / \mathrm{kg}$ group demonstrated an average SBR $(5.3 \pm 3.5)$ similar to that of the $2.5-\mathrm{mg} / \mathrm{kg}$ group $(4.5 \pm 2.1 ; \mathrm{p}=0.32)$. Tumors $\leq 10 \mathrm{~mm}$ below the cortex generated a higher SBR than deep lesions $(5.3 \pm 0.49$ vs $3.38 \pm 0.46, p=0.05)$. The following factors did not significantly affect tumor SBR: patient sex $(p=0.90)$, prior surgery $(p=0.31)$, prior radiation $(p=0.98)$, infusion to surgery time $(p=0.85)$, and tumor location $(\mathrm{p}=0.84)$.

Next, the fluorescence intensity of BMs based on the primary pathology was surveyed. Lung cancer (SBR $5.7 \pm$ 3.1), breast cancer (SBR $5.7 \pm 3.9$ ), and colon cancer (SBR $5.2 \pm 2.3$ ) demonstrated comparable fluorescence strength, whereas melanomas exhibited significantly dimmer signals (SBR $3.1 \pm 1.6$; $\mathrm{p}=0.047$ ) (Fig. 3). When evaluating the effects of ICG dose on SBR within primary tumor groups, a significant difference was noted in the lung can- 


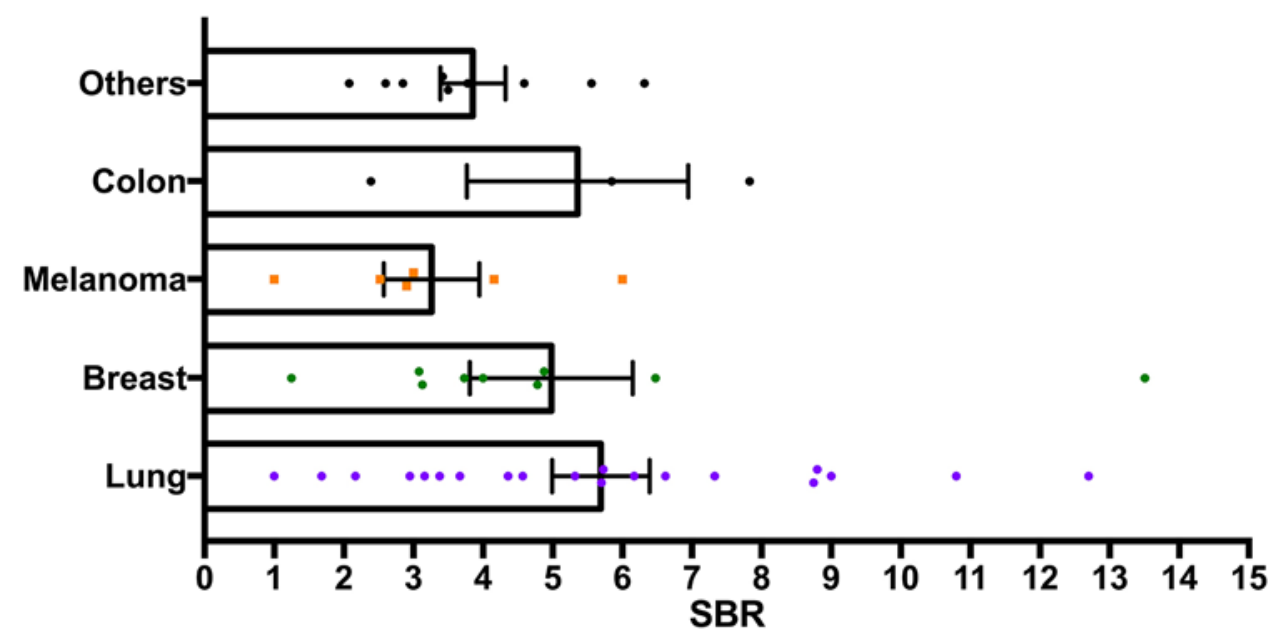

FIG. 3. Pathology-based SBR comparison: comparison of average SBRs of metastases of lung cancer, melanoma, breast cancer, colon cancer, and other pathologies. A significantly lower SBR of the melanoma pathologies $(p=0.047)$ was demonstrated; NIR fluorescence from the other tumors was not statistically different. Figure is available in color online only.

cer cohort (SBR $7.8 \pm 3.2$ in the $5-\mathrm{mg} / \mathrm{kg}$ group vs $4.6 \pm$ 2.5 in the $2.5-\mathrm{mg} / \mathrm{kg}$ group, $\mathrm{p}=0.024)$. The other primary malignancies did not show a significant SBR difference between ICG doses.

\section{Neoplastic Margin Detection Using NIR Fluorescence}

A total of 91 specimens were retrieved from 47 patients. In the operating room, specimens were coded as positive or negative based on the surgeon's impression. Fifty-nine specimens were coded as tumor positive under WL inspection, while 87 were coded as positive under NIR view. Overall, NIR fluorescence demonstrated a higher sensitivity but lower specificity than WL inspection for detecting neoplastic tissue. The PPV of NIR fluorescence was $80.5 \%$ (vs $98.3 \%$ under WL); in contrast, the NPV improved to $100 \%$ under NIR (62.5\% under WL) (Table 2).

Out of the 91 specimens, 40 were tumor margins collected after resection of the gross tumor. Test characteristics at the margins were calculated using the surgeon's tumor coding under WL and NIR (Table 3). Similar to the earlier findings, NIR fluorescence demonstrated higher sensitivity in the ability to identify tumor margins within the resection cavity (100\% vs $36.8 \%$ under WL), albeit with lower specificity (19.1\% vs $95.2 \%)$.

To improve the specificity of NIR diagnostics, the ROC curve for margin specimens was analyzed. Among different thresholds of SBR tested, the greatest accuracy was achieved with a threshold SBR of 3 rather than the sur- geon's "intraoperative assessment," which corresponded to an SBR of 2 (Table 3). With the new SBR cutoff of 3, the specificity (61.9\%), PPV (66.7\%), and accuracy (72.5\%) using SWIG markedly improved, with a slight decrease in sensitivity $(84.2 \%)$.

\section{Correlation of Postresection NIR View to Postoperative MRI}

Two patients were excluded because no postoperative MRI was performed. Overall, GTR was accomplished in $73.5 \%(36 / 49)$ of tumors. The rate of GTR was not influenced by patient age $(\mathrm{p}=0.91)$, sex $(\mathrm{p}=0.84)$, tumor location ( $p=0.43)$, or tumor laterality $(p=0.70)$. The low-dose group demonstrated a rate of GTR $(81.8 \%)$ similar to that of the high-dose group $(66.7 \% ; \mathrm{p}=0.24)$. The only statistically significant factor that influenced the rate of GTR was tumor depth: a higher GTR rate was achieved in more superficial tumors on linear regression $(p=0.040)$.

Upon completion of surgery, a final view of the operative field was captured in 44 cases. The presence and location of the residual fluorescence on the final view was examined by independent reviewers. A clean margin with minimal or no residual fluorescence was observed in 36\% (16/44) of the cases. Notably, all 16 cases had a complete tumor resection based on postoperative MRI. A significant correlation between a clean final view and GTR was confirmed by chisquare analysis $(\mathrm{p}=0.007)$. However, residual NIR fluorescence was not highly specific (47.1\%) for predicting residual gadolinium enhancement. In cases in which residual

TABLE 2. Test characteristics for all specimens using WL inspection versus intraoperative NIR imaging

\begin{tabular}{|c|c|c|c|c|c|c|c|c|c|}
\hline & \multirow{2}{*}{$\begin{array}{c}\text { Intraop } \\
\text { Visualization }\end{array}$} & \multicolumn{2}{|c|}{ Tumor Pathology } & \multirow[b]{2}{*}{ Prevalence } & \multicolumn{5}{|c|}{ Test Statistic $(95 \% \mathrm{Cl})$} \\
\hline & & Yes & No & & Sensitivity & Specificity & PPV & NPV & Accuracy \\
\hline \multirow{2}{*}{ WL } & Yes & 58 & 1 & \multirow{4}{*}{$\begin{array}{c}76.9 \% \\
(66.9 \%-85.1 \%)\end{array}$} & $82.9 \%$ & $95.2 \%$ & $98.3 \%$ & $62.5 \%$ & $85.7 \%$ \\
\hline & No & 12 & 20 & & $(72.0 \%-90.8 \%)$ & $(76.2 \%-99.9 \%)$ & $(89.5 \%-99.8 \%)$ & $(49.7 \%-73.8 \%)$ & $(76.8 \%-92.2 \%)$ \\
\hline \multirow{2}{*}{ NIR } & Yes & 70 & 17 & & $100 \%$ & $19.1 \%$ & $80.5 \%$ & $100 \%$ & $81.3 \%$ \\
\hline & No & 0 & 4 & & $(94.9 \%-100 \%)$ & $(5.5 \%-41.9 \%)$ & $(77.0 \%-83.5 \%)$ & $(100 \%-100 \%)$ & $(71.9 \%-88.7 \%)$ \\
\hline
\end{tabular}


TABLE 3. Test characteristics for margin specimens

\begin{tabular}{|c|c|c|c|c|c|c|c|c|}
\hline & \multicolumn{2}{|c|}{ Tumor Pathology } & \multirow[b]{2}{*}{ Prevalence } & \multicolumn{5}{|c|}{ Test Statistic (95\% Cl) } \\
\hline & Yes & No & & Sensitivity & Specificity & PPV & NPV & Accuracy \\
\hline \multicolumn{9}{|l|}{ WL } \\
\hline Yes & 7 & 1 & \multirow{8}{*}{$\begin{array}{c}47.5 \% \\
(31.5 \%-63.9 \%)\end{array}$} & $36.8 \%$ & $95.2 \%$ & $87.5 \%$ & $62.5 \%$ & $67.5 \%$ \\
\hline No & 12 & 20 & & $(16.3 \%-61.6 \%)$ & $(76.2 \%-99.9 \%)$ & $(48.6 \%-98.1 \%)$ & $(53.9 \%-70.4 \%)$ & $(50.9 \%-81.4 \%)$ \\
\hline NIR & & & & & & & & \\
\hline Yes & 19 & 17 & & $100 \%$ & $19.1 \%$ & $52.8 \%$ & $100 \%$ & $57.5 \%$ \\
\hline No & 0 & 4 & & $(82.4 \%-100 \%)$ & $(5.45 \%-41.9 \%)$ & $(47.6 \%-57.9 \%)$ & $(100 \%-100 \%)$ & $(40.9 \%-73.0 \%)$ \\
\hline \multicolumn{8}{|l|}{$S B R>3$} & \\
\hline Yes & 16 & 8 & & $84.2 \%$ & $61.9 \%$ & $66.7 \%$ & $81.3 \%$ & $72.5 \%$ \\
\hline No & 3 & 13 & & $(60.4 \%-96.6 \%)$ & $(38.4 \%-81.9 \%)$ & (52.9\%-78.1\%) & $(59.3 \%-92.8 \%)$ & $(56.1 \%-85.4 \%)$ \\
\hline
\end{tabular}

signals were present on both modalities, 3D image analysis demonstrated the colocalization of the residual fluorescence with gadolinium enhancement (Fig. 4).

\section{Clinical Outcomes}

Of the 47 patients, 39 received postoperative SRS and 2 received whole-brain radiotherapy (WBRT). Two patients rapidly declined and died after surgery, 2 had their care transferred to a different institution, and 2 did not receive therapy upon careful discussion on disease stabil- ity and patient preference at radiation oncology and brain tumor conferences. In 39 of 41 cases, patients received planned adjuvant radiotherapy approximately 1 month after debulking surgery; 2 patients initially refused radiation and subsequently received salvage radiotherapy upon evidence of disease recurrence. Hence, with the exception of very selective cases, the decision to administer radiation was not influenced by postoperative imaging results.

The mean PFS for the cohort was 15 months (range

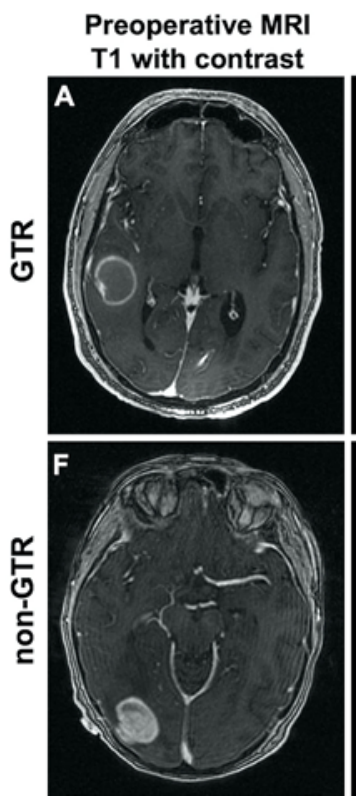

Postoperative MRI
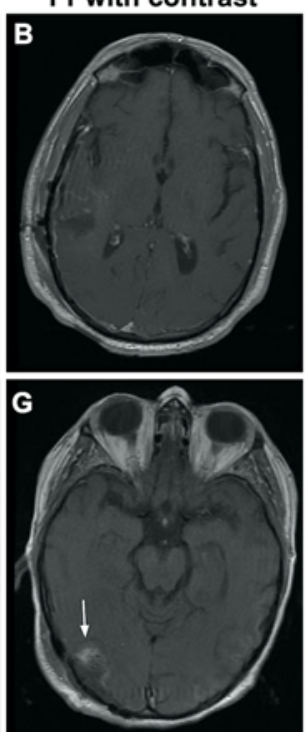

White-Light view
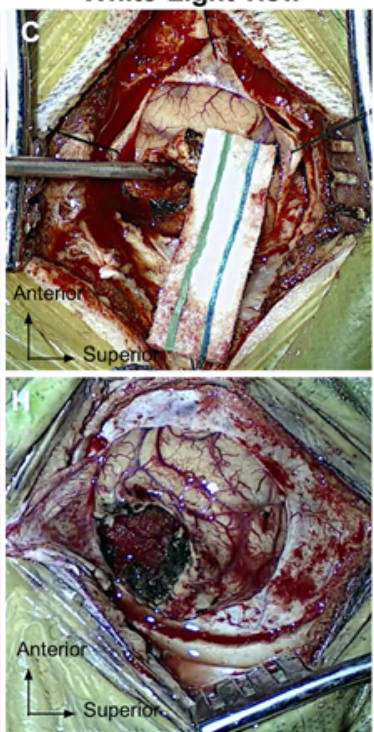

NIR view
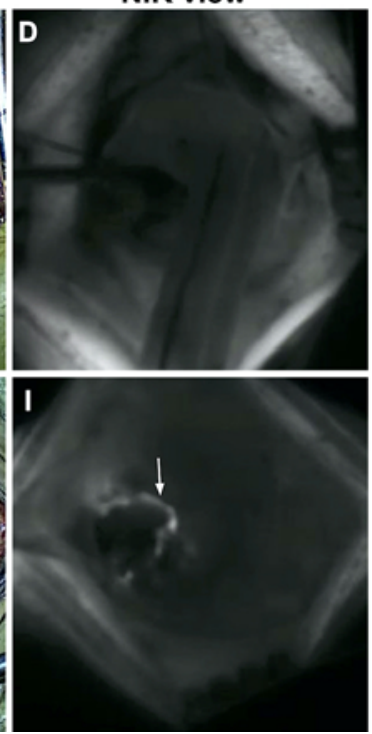

NIR pseudo-color overlay

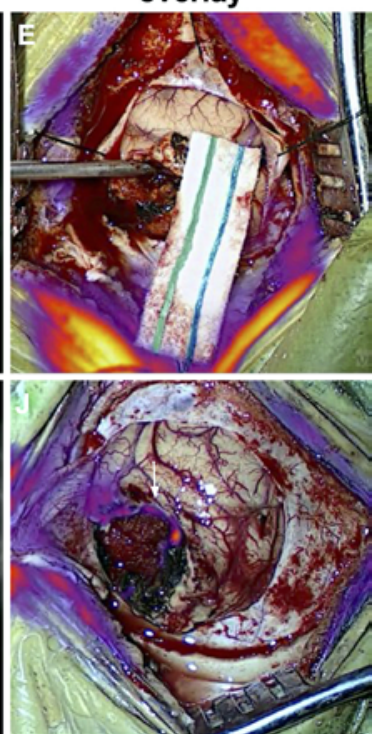

FIG. 4. Correlation between postresection NIR view with postoperative MRI. A-E: Absence of NIR fluorescence on NIR final view predicts complete resection of the enhancing tumor on postoperative MRI. Preoperative T1 MRI with gadolinium contrast $(\mathbf{A})$ demonstrating a $3.1 \times 2.9-\mathrm{cm}$ contrast-enhancing intracranial metastasis in the right temporal lobe. Postoperative T1 MRI with gadolinium contrast (B) demonstrated GTR with minimal peripheral enhancement consistent with postoperative changes. Both WL (C) and NIR (D and E) imaging of the surgical cavity after resection suggested the absence of residual neoplasm. F-J: Residual fluorescence on the NIR final view corresponds with residual contrast-enhancing tissue on postoperative MRI. Preoperative T1 MRI with gadolinium contrast $(F)$ demonstrating a $3.0 \times 2.5-\mathrm{cm}$ contrast-enhancing intracranial metastasis in the right occipital lobe. Postoperative T1 MRI with gadolinium contrast $(\mathbf{G})$ demonstrated residual nodular enhancement on the anterior surface of the resection cavity. The postresection WL view $(\mathbf{H})$ did not show evidence of residual neoplasm. NIR final view imaging revealed residual fluorescence with nodular appearance along the anterior resection cavity, corresponding with the findings on MRI (I and $\mathrm{J})$. Figure is available in color online only. 
0.7-50.7 months). PFS was $74.5 \%$ at 6 months and $40.1 \%$ at 12 months. Prior surgery on different BMs appeared to be the only statistically significant predictor for PFS ( $p=$ $0.002)$. Other variables tested include sex $(p=0.16)$, age 61 years and older $(\mathrm{p}=0.20)$, and primary pathology $(\mathrm{p}$ $=0.27$ ). All Kaplan-Meier survival curves are illustrated (Fig. 5). While primary pathology was not a significant predictor for PFS overall, patients with melanoma had enhanced PFS compared to other pathologies $(p=0.05)$. To avoid confounding bias, as melanomas behave differently under NIR imaging, melanoma patients were excluded when residual fluorescence was examined as a predictor for survival. Interestingly, in nonmelanoma patients, residual fluorescence appeared to be a better predictor for PFS than postoperative MRI. At 12 months postsurgery, PFS was $27 \%$ for patients with residual enhancement on postoperative MRI versus $29 \%$ for those with no residual enhancement; in contrast, PFS was $20 \%$ for those with residual fluorescence on the postresection NIR view versus $38 \%$ for those without residual fluorescence. These results are consistent with the systemic disease recurrence pattern. Recurrence was observed in 16 patients (34\%) prior to the last date of contact. Of these recurrence cases, residual enhancement was detected in only 8 patients on postoperative MRI, whereas residual fluorescence on the final NIR image was demonstrated in 12 patients. Otherwise, no correlation was observed between PFS and the number of metastases $(p=0.82)$, tumor location $(p=0.72)$, or prior radiation $(\mathrm{p}=0.56)$.

The mean OS for the entire cohort was 19 months (range 0.7-65.3 months). Female patients had significantly longer OS $(p=0.009)$ compared to male patients. Other factors examined in the aforementioned analyses were not statistically significantly associated with OS, including prior surgery on $\mathrm{BM}(\mathrm{p}=0.31)$.

\section{Discussion}

\section{SWIG Leads to Strong NIR Signal in Almost All Gadolinium-Enhancing BMs}

In this report, we present the final results of a prospective clinical trial evaluating intraoperative imaging and clinical outcomes in 47 patients with BMs using SWIG. Unlike 5-ALA, all BMs demonstrated strong NIR fluorescence after delayed, high-dose ICG administration. Since 5-ALA is a prodrug that is enzymatically converted to PpIX, and because the precise biological reason that some tumors accumulate PpIX is not well understood, tumor fluorescence after 5-ALA administration in BM is unpredictable. Given the histological variety of BMs, a more predictable dye is needed to image these tumors. As such, we hypothesized that SWIG could serve as an imaging adjunct during $\mathrm{BM}$ resection. In this series of 47 patients, $100 \%$ of BMs demonstrated NIR fluorescence after SWIG administration, with an average SBR of 4.9, supporting our hypothesis that BMs of varying pathologies demonstrate strong NIR fluorescence with SWIG.

We further identified predictors of fluorescence signal strength in gross tumors. First, deep lesions demonstrated lower SBR. This is likely a consequence of the normal parenchyma being closer to the camera than the tumor deep in the cavity, causing a disproportionate amount of background signal to be captured, since light intensity decreases as a factor of the distance squared. There was no significant difference in SBR between the two most common intracranial metastases (lung and breast), but melanomas did demonstrate weaker signals. We hypothesize that this may be caused by the intrinsic property of melanin pigment to block light penetration, as similar findings have been documented with 5-ALA. ${ }^{10,22,29}$ Therefore, caution should be taken when interpreting fluorescence profiles in melanoma metastases intraoperatively. In addition, while there was no significant difference in the SBR of gross tumors between the two ICG doses ( $2.5 \mathrm{vs} 5 \mathrm{mg} / \mathrm{kg}$ ), dose was a significant predictor of SBR within the lung pathology subset, suggesting that BMs from the lung are more responsive to the dose of ICG.

One advantage of NIR fluorescence is the greater depth of penetration compared to visible-light fluorescence. As such, we were able to localize $77 \%$ of the tumors through the dura and 95\% through the intact cortex, whereas 5-ALA does not reveal the tumor until it is in direct sight of the surgeon. Identification of tumor through the dura did appear to be dose dependent as $91.7 \%$ of the cases were identified at $5 \mathrm{mg} / \mathrm{kg}$ and only $53.3 \%$ at $2.5 \mathrm{mg} / \mathrm{kg}$. Nevertheless, SWIG enables surgeons to localize tumors prior to dural and cortical opening in the majority of cases, and SWIG is not subject to navigational or registration errors as seen with conventional neuronavigation systems that rely on preoperative MRI ${ }^{30}$ By identifying dye accumulation early, the dural incision can be precisely targeted to the tumor location.

\section{Quantitative Fluorescence Analysis Allows for Optimization of Margin Detection}

In our previous SWIG studies, identification of tumor under NIR fluorescence was evaluated based on the surgeon's coding in the operating room. We observed that specimens with $\mathrm{SBR} \geq 2$ were consistently designated as positive, likely due to the signal amplification of the VisionSense color overlaying algorithm. However, correspondence between true tumor pathology and a quantitative SBR cutoff value had not been defined. In this study, we found through post hoc ROC analysis that selecting an SBR of 3 as the cutoff yielded optimal test characteristics for correct tumor margin identification. We suspect that intraoperative tumor manipulation may cause ICG to leak out of the tumor into the resection cavity, causing nonspecific fluorescence of the surrounding area. Since there is evidence suggesting that ICG can be taken into tumor cells via endocytosis, it is also possible that it is the actual ICG-laden tumor cells that are leaking into brain parenchyma during surgical manipulation. Regardless, we hypothesize that raising the SBR cutoff helps to distinguish between signal from tumor and nonspecific fluorescence, leading to more accurate determination of neoplastic margins. By raising the threshold from 2 to 3 , the overall diagnostic accuracy increased from $57.5 \%$ to $72.5 \%$. Future software algorithms may be incorporated into commercial software to assist the surgeon in real time to identify optimal threshold cutoffs as increasingly more work is done in this area. 

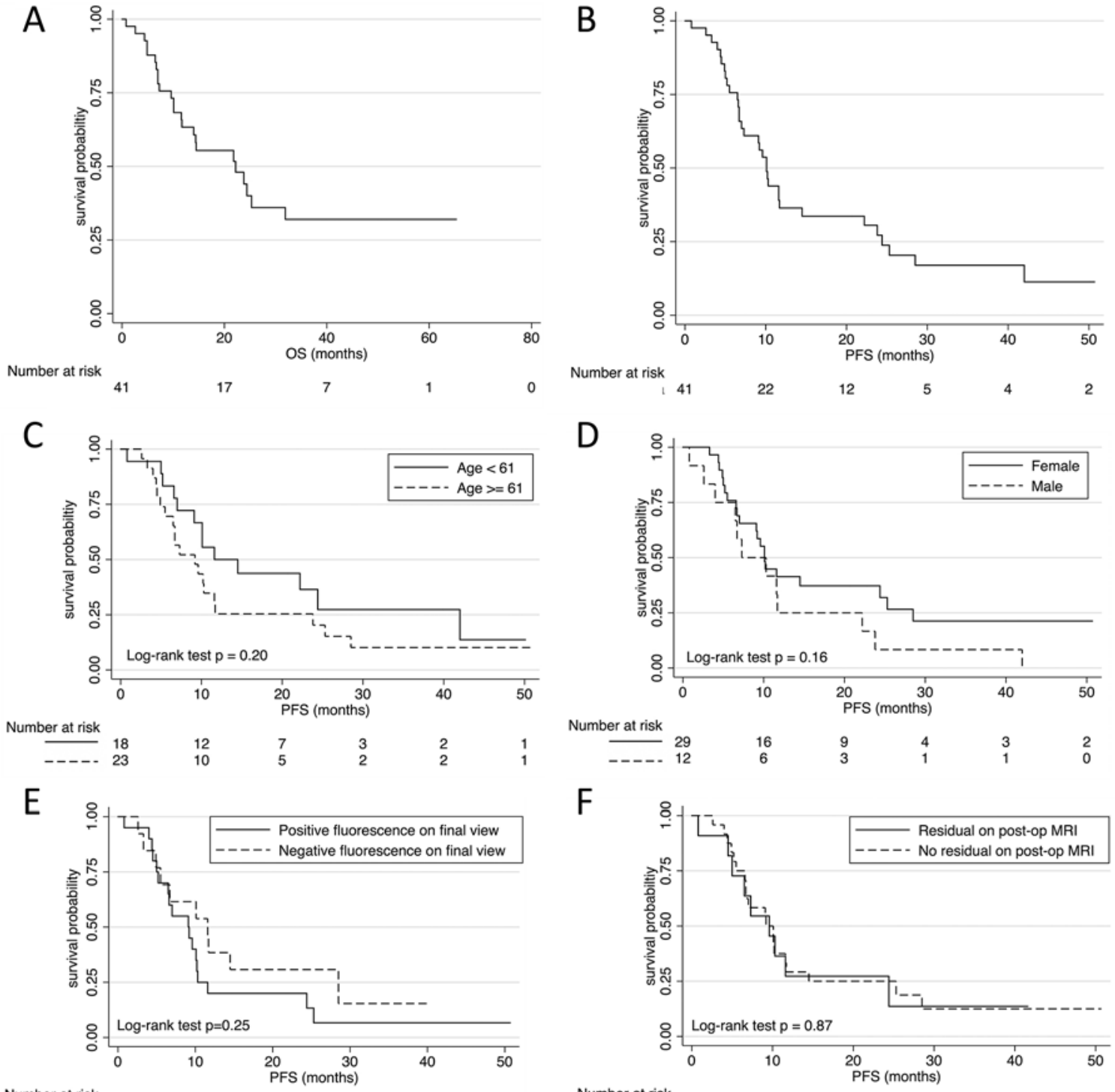

$\mathrm{F}$
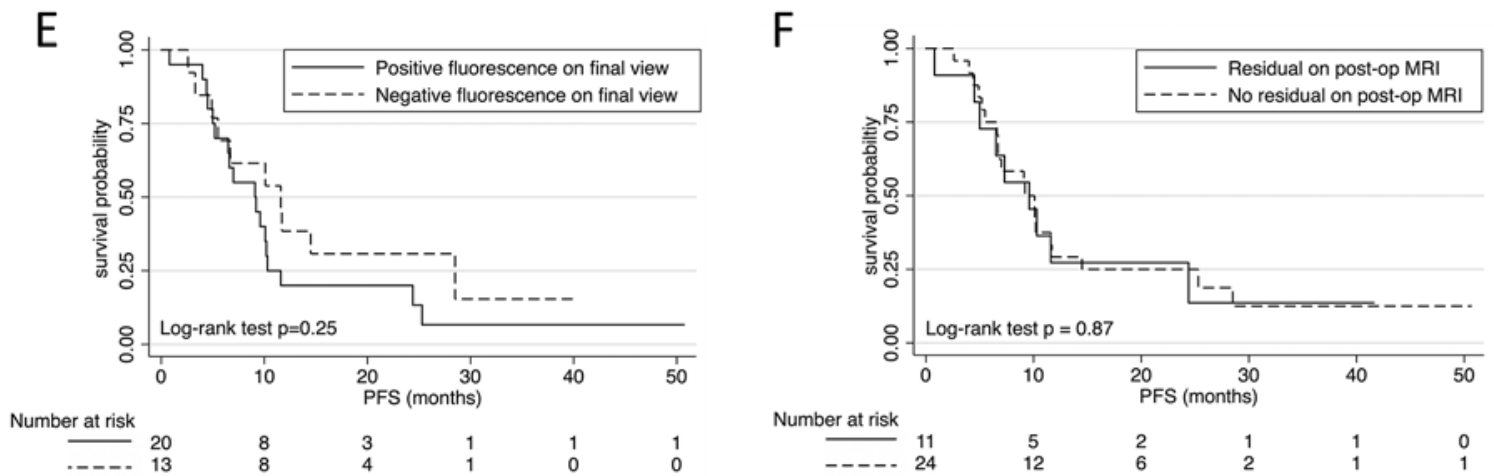

G

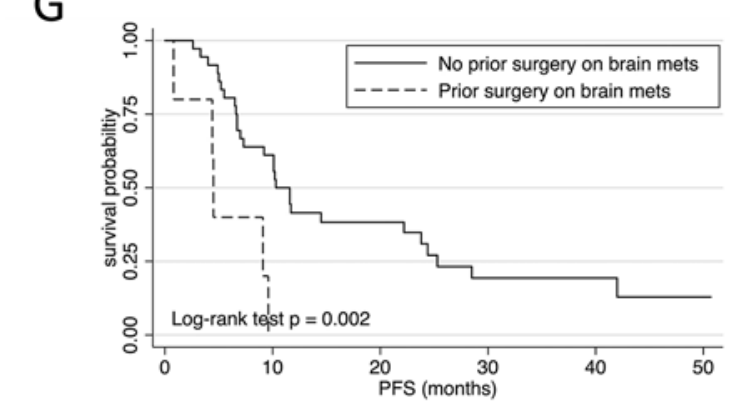

$\mathrm{H}$

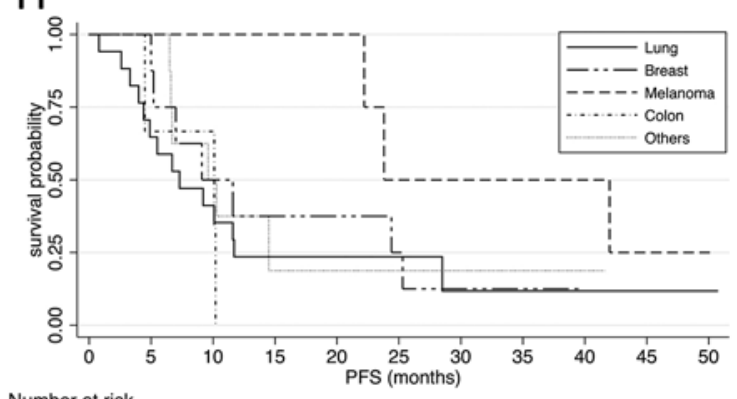

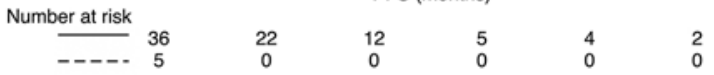

FIG. 5. Kaplan-Meier survival curves. A: OR was 19 months (range 0.7-65.3 months). B: PFS was 15 months (range 0.7-50.7 months). C-H: PFS stratified by age (C), sex (D), NIR final view (E), postoperative MRI (F), prior surgery on BM (G), and primary pathology $(\mathbf{H})$. mets $=$ metastases. 


\section{Clean NIR View of Postresection Cavity Predicts GTR}

An additional potential utility of FGS is postoperative evaluation of the extent of resection. ${ }^{31,32}$ Currently in neurosurgery, postoperative MRI is the gold standard for evaluating residual neoplasms, but it is not perfect, particularly in the immediate postoperative period. Early reactive enhancement and residual neoplasm-induced contrast enhancement can be difficult to differentiate. ${ }^{33,34}$ In our study, the radiologist reported "peripheral enhancement cases in resection cavity, small residual not excluded" in 15 (30\%), indicating a low but not absent index of suspicion. Also interesting was that there were 4 cases in which a suspected residual "nodular" neoplasm was detected on the 1-month follow-up MRI, but the immediate postoperative imaging showed little to no enhancement. Hence, it is possible that small amounts of residual neoplasm may have been mistaken as normal postoperative changes on MRI and vice versa. Indeed, precisely because it is difficult to evaluate GTR and due to the high local recurrence rate, the standard of care is to treat patients with radiation therapy or radiosurgery afterward.

Remarkably, our small study found that in nonmelanoma patients, residual fluorescence on NIR final view was a better predictor for disease recurrence and, subsequently, PFS, compared to postoperative MRI. This is consistent with our hypothesis that small residual tumors missed on MRI may have been captured by the intraoperative fluorescence. Nonetheless, there are concerns that tumors may leak dye into the resection cavity during surgery, causing nonspecific NIR fluorescence in healthy brain tissues. Hence, we think that with the current technology it is premature to alter the extent of resection based on neoplastic margin fluorescence until we find reliable ways to improve specificity. However, it is important to note that in all 16 cases in which no residual fluorescence was detected, the MRI showed the same result. This suggests that the NIR final view is at least as sensitive as MRI at detecting residual disease and may improve overall sensitivity when considered in conjunction with MRI. As such, we have an opportunity to improve determination of true GTR through adding another imaging modality, which may benefit our decisions on the adjuvant radiotherapy approach. In our study, only 2 patients did not receive postoperative radiation therapy, given careful consideration of disease stability, and both of them did not suffer from disease recurrence for the study duration. While this observation is only preliminary, we have reason to believe that with improved surgical resection and more robust imaging tools to evaluate operative results, there is hope for altering our approach to radiotherapy and avoiding the additional risks in certain cases, thereby improving survival and quality of life for patients.

\section{Study Limitations}

There are several limitations to this study. First, sensitivity and specificity are highly dependent on the number and spatial randomness of the biopsies taken, which may lead to sampling bias. Indeed, the FDA has suggested not using the terms "sensitivity" and "specificity" when discussing surgical biopsies, as these terms require random sampling, which, to avoid iatrogenic neurological deficits, is generally not done by neurosurgeons. ${ }^{35} \mathrm{~A}$ future study in which biopsies are taken more systematically in each patient may yield true independent observations and a better estimate of the test characteristics of our SWIG technique. Second, the localization of ICG in tumor tissue is not well understood. Onda et al. have demonstrated that ICG is taken up into tumor cells via endocytosis. ${ }^{36}$ Our intraoperative visualization platform does not have high enough spatial resolution to replicate the optical techniques to determine whether the NIR signal originates from the tumor cells or their extracellular matrix environment. Last, although this is a prospective cohort study, the analysis was conducted retrospectively, and we thus propose a future study that randomizes patients to surgery with ICG-derived NIR fluorescence guidance versus use of ICG but without visualization of the fluorescence at the margins. In doing so, we hope to elucidate the impact of the SWIG technique on clinical outcomes.

\section{Conclusions}

In this study, visible NIR fluorescence was detected in all 51 intracranial metastases, demonstrating the wide applicability and reliability of the high-dose, delayed ICG (SWIG) technique in surgery for patients with BMs. Specifically, this technique allows for dose-dependent, transdural localization of neoplasms and improved sensitivity in neoplastic margin detection. The reduced specificity can be improved with careful quantitative analysis of SBR, which requires a larger sample size to corroborate. Last but not least, we identified the absence of residual fluorescence in the postresection cavity as a better predictor for GTR, reduced recurrence rate, and improved PFS compared to postoperative MRI. This suggests that intraoperative NIR imaging with SWIG may prove useful in evaluating the extent of resection in conjunction with MRI and guide decisions on intracranial metastasis management.

\section{Acknowledgments}

Supported in part by the Institute for Translational Medicine and Therapeutics of the Perelman School of Medicine at the University of Pennsylvania (J.Y.K.L.). In addition, research reported in this publication was supported by the National Center for Advancing Translational Sciences of the National Institutes of Health under award numbers UL1TR000003 (J.Y.K.L.) and TL1TR001880 (S.S.C.). The content is solely the responsibility of the authors and does not necessarily represent the official views of the NIH.

\section{References}

1. Dagogo-Jack I, Carter SL, Brastianos PK. Brain metastasis: clinical implications of branched evolution. Trends Cancer. 2016;2(7):332-337.

2. Ferguson SD, Wagner KM, Prabhu SS, et al. Neurosurgical management of brain metastases. Clin Exp Metastasis. 2017; 34(6-7):377-389.

3. Lowery FJ, Yu D. Brain metastasis: unique challenges and open opportunities. Biochim Biophys Acta Rev Cancer. 2017; 1867(1):49-57.

4. Nayak L, Lee EQ, Wen PY. Epidemiology of brain metastases. Curr Oncol Rep. 2012;14(1):48-54.

5. Prabhu RS, Miller KR, Asher AL, et al. Preoperative stereotactic radiosurgery before planned resection of brain metasta- 
ses: updated analysis of efficacy and toxicity of a novel treatment paradigm. J Neurosurg. 2019;131(5):1387-1394.

6. Patchell RA, Tibbs PA, Regine WF, et al. Postoperative radiotherapy in the treatment of single metastases to the brain: a randomized trial. JAMA. 1998;280(17):1485-1489.

7. Graber JJ, Cobbs CS, Olson JJ. Congress of Neurological Surgeons systematic review and evidence-based guidelines on the use of stereotactic radiosurgery in the treatment of adults with metastatic brain tumors. Neurosurgery. 2019; 84(3):E168-E170.

8. Brastianos PK, Curry WT, Oh KS. Clinical discussion and review of the management of brain metastases. J Natl Compr Canc Netw. 2013;11(9):1153-1164.

9. Byun YH, Gwak HS, Kwon J-W, et al. Local recurrence of brain metastasis reduced by intra-operative hyperthermia treatment. Int J Hyperthermia. 2019;35(1):168-175.

10. Lee JYK, Pierce JT, Zeh R, et al. Intraoperative near-infrared optical contrast can localize brain metastases. World Neurosurg. 2017;106:120-130.

11. Minniti G, Clarke E, Lanzetta G, et al. Stereotactic radiosurgery for brain metastases: analysis of outcome and risk of brain radionecrosis. Radiat Oncol. 2011;6:48.

12. Kocher M, Soffietti R, Abacioglu U, et al. Adjuvant wholebrain radiotherapy versus observation after radiosurgery or surgical resection of one to three cerebral metastases: results of the EORTC 22952-26001 study. J Clin Oncol. 2011;29(2): $134-141$.

13. Williams BJ, Suki D, Fox BD, et al. Stereotactic radiosurgery for metastatic brain tumors: a comprehensive review of complications. J Neurosurg. 2009;111(3):439-448.

14. Burt M, Wronski M, Arbit E, Galicich JH. Resection of brain metastases from non-small-cell lung carcinoma. Results of therapy. J Thorac Cardiovasc Surg. 1992;103(3):399-411.

15. Churilla TM, Chowdhury IH, Handorf E, et al. Comparison of local control of brain metastases with stereotactic radiosurgery vs surgical resection: a secondary analysis of a randomized clinical trial. JAMA Oncol. 2019;5(2):243-247.

16. Kamp MA, Munoz-Bendix C, Mijderwijk H-J, et al. Is 5-ALA fluorescence of cerebral metastases a prognostic factor for local recurrence and overall survival? J Neurooncol. 2019;141(3):547-553.

17. Motekallemi A, Jeltema H-R, Metzemaekers JDM, et al. The current status of 5-ALA fluorescence-guided resection of intracranial meningiomas-a critical review. Neurosurg Rev. 2015;38(4):619-628.

18. Zhang DY, Singhal S, Lee JYK. Optical principles of fluorescence-guided brain tumor surgery: a practical primer for the neurosurgeon. Neurosurgery. 2019;85(3):312-324.

19. Ferraro N, Barbarite E, Albert TR, et al. The role of 5-aminolevulinic acid in brain tumor surgery: a systematic review. Neurosurg Rev. 2016;39(4):545-555.

20. Schatlo B, Stockhammer F, Barrantes-Freer A, et al. 5-Aminolevulinic acid fluorescence indicates perilesional brain infiltration in brain metastases. World Neurosurg X. 2019;5: 100069.

21. Marbacher S, Klinger E, Schwyzer L, et al. Use of fluorescence to guide resection or biopsy of primary brain tumors and brain metastases. Neurosurg Focus. 2014;36(2):E10.

22. Marhold F, Mercea PA, Scheichel F, et al. Detailed analysis of 5 -aminolevulinic acid induced fluorescence in different brain metastases at two specialized neurosurgical centers: experience in 157 cases. J Neurosurg. 2020;133(4):1032-1043.

23. Okuda T, Kataoka K, Yabuuchi T, et al. Fluorescence-guided surgery of metastatic brain tumors using fluorescein sodium. $J$ Clin Neurosci. 2010;17(1):118-121.

24. Höhne J, Hohenberger C, Proescholdt M, et al. Fluorescein sodium-guided resection of cerebral metastases-an update. Acta Neurochir (Wien). 2017;159(2):363-367.
25. Schebesch K-M, Hoehne J, Hohenberger C, et al. Fluorescein sodium-guided resection of cerebral metastases-experience with the first 30 patients. Acta Neurochir (Wien). 2015;157(6): 899-904.

26. Cho SS, Zeh R, Pierce JT, et al. Comparison of near-infrared imaging camera systems for intracranial tumor detection. Mol Imaging Biol. 2018;20(2):213-220.

27. DSouza AV, Lin H, Henderson ER, et al. Review of fluorescence guided surgery systems: identification of key performance capabilities beyond indocyanine green imaging. $J$ Biomed Opt. 2016;21(8):80901.

28. Lin NU, Lee EQ, Aoyama H, et al. Response assessment criteria for brain metastases: proposal from the RANO group. Lancet Oncol. 2015;16(6):e270-e278.

29. Ando H, Ohagi Y, Yoshida M, et al. Melanin pigment interrupts the fluorescence staining of mitochondria in melanocytes. J Dermatol Sci. 2016;84(3):349-351.

30. Cho SS, Teng CW, Ramayya A, et al. Surface-registration frameless stereotactic navigation is less accurate during prone surgeries: intraoperative near-infrared visualization using second window indocyanine green offers an adjunct. Mol Imaging Biol. 2020;22(6):1572-1580.

31. Predina JD, Newton AD, Corbett C, et al. A clinical trial of TumorGlow to identify residual disease during pleurectomy and decortication. Ann Thorac Surg. 2019;107(1):224-232.

32. Holt D, Parthasarathy AB, Okusanya O, et al. Intraoperative near-infrared fluorescence imaging and spectroscopy identifies residual tumor cells in wounds. J Biomed Opt. 2015; 20(7):76002.

33. Lescher S, Schniewindt S, Jurcoane A, et al. Time window for postoperative reactive enhancement after resection of brain tumors: less than 72 hours. Neurosurg Focus. 2014; 37(6):E3

34. Kim HY, Kim ST, Kim H-J, et al. Differentiation of postoperative changes and residual tumors in dynamic contrast-enhanced sella MRI after transsphenoidal resection of pituitary adenoma. Medicine (Baltimore). 2019;98(27):e16089.

35. Tummers WS, Warram JM, Tipirneni KE, et al. Regulatory aspects of optical methods and exogenous targets for cancer detection. Cancer Res. 2017;77(9):2197-2206.

36. Onda N, Kimura M, Yoshida T, Shibutani M. Preferential tumor cellular uptake and retention of indocyanine green for in vivo tumor imaging. Int J Cancer. 2016;139(3):673-682.

\section{Disclosures}

The authors report no conflict of interest concerning the materials or methods used in this study or the findings specified in this paper.

\section{Author Contributions}

Conception and design: Lee, Teng. Acquisition of data: Lee, Teng, Singh, Somers, Buch. Analysis and interpretation of data: Teng, Cho. Drafting the article: Teng. Critically revising the article: Lee, Teng, Cho, De Ravin. Reviewed submitted version of manuscript: Lee, Teng, Cho. Approved the final version of the manuscript on behalf of all authors: Lee. Statistical analysis: Teng, Singh. Study supervision: Lee, Brem, Singhal, Delikatny.

\section{Supplemental Information \\ Videos}

Video 1. https://vimeo.com/458241710.

\section{Correspondence}

John Y. K. Lee: Hospital of the University of Pennsylvania, Philadelphia,PA.leejohn@pennmedicine.upenn.edu. 